\title{
Molecular characterization of Prototheca strains isolated from Italian dairy herds
}

\author{
M. Ricchi, ${ }^{*}$ M. Goretti,† E. Branda,† G. Cammi, ${ }^{*}$ C. A. Garbarino, ${ }^{*}$ B. Turchetti,† P. Moroni, $\$ \S$ \\ N. Arrigoni, ${ }^{*}$ and P. Buzzini ${ }^{1}$ \\ *Istituto Zooprofilattico Sperimentale of Lombardia and Emilia Romagna, I-29027 Piacenza, Italy \\ †Department of Applied Biology and Industrial Yeasts Collection DBVPG, University of Perugia, I-06121 Perugia, Italy \\ ‡Quality Milk Production Services, Cornell University, Ithaca, NY 14850 \\ §Università degli Studi di Milano, Department of Veterinary Pathology, Hygiene and Public Health, I-20133 Milan, Italy
}

\section{ABSTRACT}

One hundred sixty-one Prototheca spp. strains isolated from composite milk and barn-surrounding environmental samples (bedding, feces, drinking, or washing water, surface swabs) of 24 Italian dairy herds were characterized by genotype-specific PCR analysis. Overall, 97.2\% of strains isolated from composite milk samples were characterized as Prototheca zopfii genotype 2, confirming its role as the main mastitis pathogen, whereas Prototheca blaschkeae was only sporadically isolated (2.8\%). Regarding environmental sampling, $84.9 \%$ of isolates belonged to $P$. zopfii genotype $2,13.2 \%$ to $P$. blaschkeae, and $1.9 \%$ to $P$. zopfii genotype 1 . The data herein contradict previous hypotheses about the supposed exclusive role of $P$. zopfii genotype 2 as the causative agent of protothecal mastitis and, on the contrary, confirm the hypothesis that such pathology could be caused by $P$. blaschkeae in a few instances.

Key words: Prototheca zopfii genotype, Prototheca blaschkeae, genotype-specific PCR analysis, milk

\section{INTRODUCTION}

The genus Prototheca includes unicellular yeast-like, colorless microalgae (phylogenetically related to Chlorella), belonging, to date, to 5 accepted species (Prototheca zopfii, Prototheca blaschkeae, Prototheca stagnora, Prototheca whickeramii, and Prototheca ulmea; Pore, 1998; Roesler et al., 2006). The presence of a sixth species (Prototheca moriformis) is still under debate (Ueno et al., 2003), and recently Satoh et al. (2010) proposed a new species, Prototheca cutis sp. nov. These microorganisms exhibit exclusively an asexual reproduction by cytoplasm splitting and the consequent formation from 2 to 16 daughter cells. Prototheca zopfii, P. stagnora, P. whickeramii, and P. ulmea are commonly associated

Received February 17, 2010.

Accepted June 25, 2010.

${ }^{1}$ Corresponding author: pbuzzini@unipg.it with some natural habitats (especially wet environments containing rotting organic matter; Pore, 1998). On the other hand, P. blaschkeae, P. whickeramii, and $P$. cutis have been associated with human protothecosis (Pore, 1998; Roesler et al., 2006, Lass-Flörl and Mayr, 2007; Satoh et al., 2010).

Although infections caused by $P$. zopfii have been observed in small animals (Ribeiro et al., 2009), mastitis in dairy cows represents the most frequent (and often dramatic) form of protothecosis in animals (Janosi et al., 2001). Mammary gland infections caused by $P$. zopfii are rarely associated with clinical signs. Accordingly, the detection of individual cases of protothecal mastitis in some herds can indicate a serious problem affecting a significant percentage of cows. Cattle appear to be susceptible to infections in all stages of lactation, including the dry period (Furuoka et al., 1989). Some authors report a particular susceptibility to the natural infection in the first few weeks of lactation (Janosi et al., 2001). In cows, the infection may be restricted to the udder or disseminated to the lymph nodes (Mc Donald et al., 1984).

Protothecal mastitis is rapidly becoming a global problem (Lagneau, 1996; Castagna de Vargas et al., 1998; Janosi et al., 2001; Buzzini et al., 2004; Scaccabarozzi et al., 2008). Studies carried out over the last 10 yr report that only a few antibiotic drugs (e.g., polyenes) or antiseptics (e.g., sodium hypochlorite, iodine compounds, indole-3-acetic acid + horseradish peroxidase, bovine lactoferrin hydrolysate, synthetic flavonoid derivatives) exhibit in vitro activity against these yeast-like microalgae (Segal et al., 1976; Casal and Gutierrez, 1983; Shahan and Pore, 1991; Marques et al., 2006; Kawai et al., 2007; Buzzini et al., 2008a,b; Tortorano et al., 2008; Cunha et al., 2010; Salerno et al., 2010). On the contrary, their effect in vivo has never been demonstrated.

In recent years, based on the results of a PCR assay carried out on P. zopfii isolates, Roesler et al. (2006) described the existence of 2 distinct $P$. zopfii genotypes. 
Moreover, Möller et al. (2007) postulated the nonpathogenic nature of both $P$. zopfii genotype 1 and $P$. blaschkeae (previously defined as $P$. zopfii genotype 3 ). In this background, the same authors suggested that $P$. zopfii genotype 2 could be the exclusive causative agent of bovine protothecal mastitis. Accordingly, P. zopfii genotype 2 was preliminarily designated as $P$. zopfii ssp. bovimastidogenes.

However, further studies only partially confirmed this assumption. A large-scale screening survey carried out on Prototheca spp. Japanese strains (isolated from bovine mastitis and surrounding cow barns) found that all isolates from mastitic milk belonged to $P$. zopfii genotype 2 (Osumi et al., 2008), whereas Marques et al. (2008) reported a few bovine mastitis associated with $P$. blaschkeae. Accordingly, these results present a complicated scenario about the occurrence of protothecal mastitis caused by $P$. zopfii genotype 2 or by $P$. blaschkeae.

The management measures applied to protothecal mastitis outbreaks are directed to control the possible sources of infection. Because of the double nature of the infection, both contagious and environmental, these measures mainly involve separate milking of infected cows, culling infected animals, and controlling environmental contamination. Accordingly, the discrimination between pathogenic and nonpathogenic species is critical in evaluating the possible sources of infection.

The aim of the study was to give a molecular characterization of Prototheca strains isolated from composite milk of cows and from environmental barn-surrounding samples, to yield a profile of the occurrence of $P$. zopfii (genotype 1 and 2) and $P$. blaschkeae in Italian dairy herds.

\section{MATERIALS AND METHODS}

\section{Sample Collection}

The number of herds (24) was chosen to represent dairies participating in a somatic cell count control project (Milk Quality Premium, Modena and Piacenza, Italy) with the problem of high SCC in the bulk tank. Samples used in this study derived from dairy herds with contagious and environmental mastitis; 18 were positive also for Prototheca and 6 negative for Prototheca.

Samples were collected from cows with clinical mastitis or high SCC and that were not responding to mastitis therapy. Infection status was defined according to the procedures recommended by the National Mastitis Council (NMC, 1999), including Prototheca. In all cases of positive diagnosis of protothecal infection, further analysis was performed on all lactating cows of the herd. As a negative control, all animals of 6 uninfected herds were sampled. The total of composite milk samples from both infected and uninfected herds was 3,208 .

Moreover, 411 environmental samples were collected from the same herds and screened for the presence of Prototheca spp. To prevent cross-contamination, all environmental samples from different sites (in both the lactating and dry cow areas) were aseptically collected in sterile vials or bags, stored at $4^{\circ} \mathrm{C}$ in a cool box, and transported within a few hours to the laboratory for microbiological analysis. Environmental samples included cow drinking water, wash water from the milk transport system and refrigeration tank, cow resting areas (bedding material), cattle feces (direct rectal sampling by single-use sleeves), and swabs samples collected from liners and in the barns.

\section{Isolation of Prototheca spp. Strains}

All microbiological substrates were from Difco (Franklin Lakes, NJ), whereas all chemicals were from Sigma (St. Louis, MO) and Fermentas (Glen Burnie, $\mathrm{MD})$.

Direct streaking of $0.01 \mathrm{~mL}$ of milk on Petri dishes containing Prototheca isolation medium (PIM) agar (Pore, 1973), blood agar, and Gassner agar was used for isolating Prototheca spp. strains from milk samples.

The isolation of Prototheca spp. strains from environmental samples was carried out by a preliminary enrichment on liquid PIM, followed by streaking on Petri dishes containing PIM agar. Colonies exhibiting a yeast-like morphology were picked and sub-cultured on yeast extract-peptone-glucose (YEPG) agar (yeast extract $10 \mathrm{~g} / \mathrm{L}$, peptone $10 \mathrm{~g} / \mathrm{L}$, glucose $20 \mathrm{~g} / \mathrm{L}$, agar 20 g/L; pH 6.5). All Prototheca spp. isolates were identified by auxonographical and biochemical methods (Pore, 1998; Roesler et al., 2003, 2006; Osumi et al., 2008).

All strains used in the present study are deposited either in the Industrial Yeasts Collection DBVPG of the University of Perugia (Italy; www.agr.unipg.it/ dbvpg) or in the collection of the Istituto Zooprofilattico Sperimentale of Lombardia and Emilia Romagna, Piacenza, Italy.

\section{DNA Extraction}

One hundred sixty-one representative Prototheca strains were analyzed: 108 from composite milk and 53 from environmental samples (bedding $=18$ strains, feces $=3$, drinking or washing water $=14$, swabs $=$ 18).

A procedure previously used for DNA extraction from yeasts (Turchetti et al., 2008) was modified for Prototheca species strains. Disruption of the cell wall was achieved by suspending 3 loopfuls of 48 -h cells (grown 
Table 1. Genotype-specific PCR primers used in this study

\begin{tabular}{|c|c|c|}
\hline Primer & Target & Sequence \\
\hline Proto $18-4 \mathrm{f}$ & Internal control for PCR & 5'-GACATGGCGAGGATTGACAGA-3' \\
\hline Proto $18-4 \mathrm{r}$ & Internal control for PCR & 5'-AGCACACCCAATCGGTAGGA-3' \\
\hline PZGT 3-IK/f & Internal control for PCR & 5'-CAGGGTTCGATTCCGGAGAG-3' \\
\hline PZGT 3-IK/R & Internal control for PCR & 5'-GAATTACCGCGGCTGCTGG-3' \\
\hline PZGT $1 / \mathrm{r}^{1}$ & Genotype 1-specific PCR & 5'-GCCAAGGCCCCCCGAAG-3' \\
\hline PZGT $2 / \mathrm{r}^{1}$ & Genotype 2-specific PCR & 5'-GTCGGCGGGGCAAAAGC-3' \\
\hline PZGT $3 / \mathrm{r}^{1}$ & Genotype 3-specific PCR & 5'-GTTGGCCCGGCATCGCT-3' \\
\hline
\end{tabular}

${ }^{1}$ These specific reverse primers were used in association with specific oligonucleotides (which also acted as part of the internal amplification controls): Proto 18-4f for the primers PZGT 1/r and PZGT 2/r; PZGT 3-IK/f for the primer PZGT 3/r.

in YEPG agar) in $500 \mu \mathrm{L}$ of lysis buffer (Tris-HCl 50 $\mathrm{m} M$, EDTA $50 \mathrm{~m} M, \mathrm{NaCl} 250 \mathrm{~m} M$, SDS $0.3 \%$ wt/vol, $\mathrm{pH}$ 8.0) and $500 \mu \mathrm{L}$ of phenol:chloroform mixture (1:1, $\mathrm{pH}=8.0$ ). One hundred fifty microliters (calculated as equivalent volume) of glass beads (diameter $=425-600$ $\mu \mathrm{m})$ was added. After centrifugation $(17,000 \times g$ for 30 min), $400 \mu \mathrm{L}$ of supernatant was mixed with an equal volume of ice-cold $99 \%$ ethanol. After precipitation, DNA was harvested by centrifugation $(12,000 \times g$ for 15 min at $4^{\circ} \mathrm{C}$ ), resuspended in $100 \mu \mathrm{L}$ of pure (analytical grade) water (Fermentas) and incubated $\left(37^{\circ} \mathrm{C}\right.$ for 30 min) with $4 \mu \mathrm{L}$ of RNase (Sigma; 93 units/mg of protein). After further precipitation with $11 \mu \mathrm{L}$ of 3 $M$ sodium acetate and $200 \mu \mathrm{L}$ of $99 \%$ ice-cold ethanol, DNA was harvested by centrifugation $(12,000 \times g$ for $15 \mathrm{~min}$ at $4^{\circ} \mathrm{C}$ ) and resuspended in $100 \mu \mathrm{L}$ of pure (analytical-grade) water.

\section{Genotype-Specific PCR Reaction}

After DNA extraction, the 108 Prototheca spp. strains were analyzed by a modified genotype-specific PCR procedure (Roesler et al., 2006). Genotype-specific primers are listed in Table 1. All PCR reactions were performed in $25-\mu \mathrm{L}$ reaction volumes containing $1 \times \mathrm{PCR}$ buffer, $2 \mathrm{mM} \mathrm{MgCl}, 250 \mu M$ of each of the 4 dNTPs, $0.8 \mu M$ of primer, and $0.02 \mathrm{U} / \mu \mathrm{L}$ of $T a q$ DNA polymerase (Fermentas).

Amplification of DNA was performed in a $\mathrm{T}$ personal Combi Thermal Cycler (Biometra GmbH, Goettingen, Germany). The P. zopfii genotype 1- and 2-specific PCR program was (1) initial denaturing step at $94^{\circ} \mathrm{C}$ for 4.5 min; (2) 30 cycles of $30 \mathrm{~s}$ at $94^{\circ} \mathrm{C}, 30 \mathrm{~s}$ at $65^{\circ} \mathrm{C}$, and $40 \mathrm{~s}$ at $72^{\circ} \mathrm{C}$; and (3) final extension step at $72^{\circ} \mathrm{C}$ for $5 \mathrm{~min}$. The P. blaschkeae-specific PCR program was (1) initial denaturing step at $94^{\circ} \mathrm{C}$ for $4.5 \mathrm{~min}$; (2) 35 cycles of 30 $\mathrm{s}$ at $94^{\circ} \mathrm{C}, 30 \mathrm{~s}$ at $63^{\circ} \mathrm{C}$, and $40 \mathrm{~s}$ at $72^{\circ} \mathrm{C}$; and (3) final extension step at $72^{\circ} \mathrm{C}$ for $5 \mathrm{~min}$. A negative control was included in all $\mathrm{PCR}$ reactions.

Amplification products were analyzed (after staining with ethidium bromide) by electrophoresis on $1.6 \%$ (wt/ vol) agarose gel. A molecular size marker (Gene Ruler 50 bp DNA ladder, Fermentas) was used for reference.

For comparative purposes, the strains $P$. zopfii genotype 1 SAG 2063 and P. blaschkeae SAG 2064, both obtained from Sammlung von Algenkulturen der Universität Göttingen, Göttingen, Germany (Roesler et al., 2006), and P. zopfii 219509/2, attributed to genotype 2 after auxonographical, biochemical, and sequencing analysis (our unpublished data), were used as control strains. No discrepant results were observed in repeated experiments.

\section{Statistical Evaluation of Data}

Correlation between the percentage of milk and environmental samples positive for Prototheca spp. was calculated by using the nonparametric Spearman rank correlation test (Myers and Well, 2003).

\section{RESULTS AND DISCUSSION}

Two-hundred thirty-nine and 69 Prototheca spp. strains were isolated from 3,208 individual milk samples and 411 environmental samples, respectively. Among them, a representative subset of strains (108 and 53 strains, respectively) was analyzed by genotype-specific PCR procedure. The percentage of milk and environmental samples positive for Prototheca spp., together with the results of genotype-specific PCR analysis on Prototheca spp. isolates, are reported (herd by herd) in Table 2.

When primers Proto 18-4f and Proto 18-4r were used, the 450-bp fragment of the internal amplification control, specific for Prototheca genus, was detected in all investigated strains (Figure 1). On the contrary, the presence of the $P$. zopfii genotype-1-specific amplicon (150 bp; Figure 1) was detected in only one strain isolated from drinking water (Table 2).

The $P$. zopfii genotype-2 pattern (165 bp; Figure 1) was observed in $97.2 \%$ of milk samples and in $84.9 \%$ of environmental samples analyzed (Table 2 ). When prim- 
Table 2. Prevalence of cows infected by Prototheca spp. in all sampled herds, number of environmental samples positive for Prototheca spp., and results of genotype-specific PCR analysis on Prototheca spp. isolates

\begin{tabular}{|c|c|c|c|c|c|c|c|c|c|c|}
\hline $\begin{array}{l}\text { Herd } \\
\text { code }\end{array}$ & $\begin{array}{l}\text { Lactating } \\
\text { cows }(\mathrm{n})\end{array}$ & $\begin{array}{l}\text { Positive } \\
\text { milk } \\
\text { samples } \\
(\mathrm{n})\end{array}$ & $\begin{array}{l}\text { Prevalence } \\
\quad(\%)\end{array}$ & $\begin{array}{c}\text { Typed } \\
\text { strains } \\
(\text { milk }) \\
(\mathrm{n})\end{array}$ & $\begin{array}{l}\text { Typing results by } \\
\text { genotype-specific } \\
\text { PCR }\end{array}$ & $\begin{array}{c}\text { Total } \\
\text { Environmental } \\
\text { samples } \\
(\mathrm{n})\end{array}$ & $\begin{array}{c}\text { Positive } \\
\text { environmental } \\
\text { samples } \\
\text { (n) }\end{array}$ & $\begin{array}{l}\text { Positive } \\
\text { samples } \\
(\%)\end{array}$ & $\begin{array}{c}\text { Positive } \\
\text { typed strains } \\
\text { (environment) } \\
\text { (n) }\end{array}$ & $\begin{array}{l}\text { Typing results by } \\
\text { genotype-specific } \\
\text { PCR }\end{array}$ \\
\hline 1 & 69 & 21 & 30.4 & 5 & P. zopfii genotype 2 & 29 & 7 & 24.1 & 5 & P. zopfii genotype 2 \\
\hline 2 & 232 & 29 & 12.5 & 22 & $\begin{array}{l}\text { P. zopfii genotype } 2(\mathrm{n}=19) \\
\text { P. blaschkeae }(\mathrm{n}=3)\end{array}$ & 38 & 2 & 5.3 & 1 & P. zopfii genotype 2 \\
\hline 3 & 362 & 11 & 3.0 & 7 & P. zopfii genotype 2 & 20 & 10 & 50.0 & 2 & P. zopfii genotype 2 \\
\hline 4 & 325 & 13 & 4.0 & 8 & P. zopfii genotype 2 & 8 & 2 & 25.0 & 1 & P. zopfii genotype 2 \\
\hline 5 & 118 & 22 & 18.6 & 2 & P. zopfii genotype 2 & 20 & 5 & 25.0 & 4 & P. zopfii genotype 2 \\
\hline 6 & 340 & 69 & 20.2 & 3 & P. zopfii genotype 2 & 37 & 6 & 16.2 & 4 & P. zopfii genotype 2 \\
\hline 7 & 140 & 2 & 1.4 & 2 & P. zopfii genotype 2 & 16 & 3 & 18.8 & 2 & P. zopfii genotype 2 \\
\hline 8 & 167 & 9 & 5.3 & 2 & P. zopfii genotype 2 & 10 & 0 & 0 & 0 & 0 \\
\hline 9 & 33 & 2 & 6.1 & 2 & P. zopfii genotype 2 & 5 & 1 & 20.0 & 1 & P. zopfii genotype 2 \\
\hline 10 & 42 & 2 & 4.8 & 2 & P. zopfii genotype 2 & 6 & 1 & 16.7 & 1 & P. zopfii genotype 2 \\
\hline 11 & 86 & 5 & 5.8 & 5 & P. zopfii genotype 2 & 5 & 1 & 20.0 & 1 & P. zopfii genotype 2 \\
\hline 12 & 54 & 3 & 5.5 & 3 & P. zopfii genotype 2 & 6 & 0 & 0 & 0 & 0 \\
\hline 13 & 65 & 5 & 7.7 & 5 & P. zopfii genotype 2 & 12 & 0 & 0 & 0 & 0 \\
\hline 14 & 95 & 12 & 12.6 & 12 & P. zopfii genotype 2 & 4 & 1 & 25.0 & 1 & P. zopfii genotype 2 \\
\hline 15 & 490 & 14 & 2.9 & 9 & P. zopfii genotype 2 & 85 & 12 & 14.1 & 12 & $\begin{array}{l}\text { P. zopfii genotype } 1(\mathrm{n}=1) \\
P . \text { zopfii genotype } 2(\mathrm{n}=9) \\
P . \text { blaschkeae }(\mathrm{n}=2)\end{array}$ \\
\hline 16 & 275 & 12 & 4.4 & 11 & P. zopfii genotype 2 & 51 & 10 & 19.6 & 10 & $\begin{array}{l}\text { P. zopfii genotype } 2(\mathrm{n}=7) \\
\text { P. blaschkeae }(\mathrm{n}=3)\end{array}$ \\
\hline 17 & 41 & 3 & 7.3 & 3 & P. zopfii genotype 2 & 15 & 2 & 13.3 & 2 & P. zopfii genotype 2 \\
\hline 18 & 55 & 5 & 9.1 & 5 & P. zopfii genotype 2 & 14 & 6 & 42.9 & 6 & $\begin{array}{l}\text { P. zopfii genotype } 2(\mathrm{n}=4) \\
P . \text { blaschkeae }(\mathrm{n}=2)\end{array}$ \\
\hline 19 & 45 & 0 & 0 & 0 & 0 & 4 & 0 & 0 & 0 & 0 \\
\hline 20 & 33 & 0 & 0 & 0 & 0 & 2 & 0 & 0 & 0 & 0 \\
\hline 21 & 38 & 0 & 0 & 0 & 0 & 6 & 0 & 0 & 0 & 0 \\
\hline 22 & 24 & 0 & 0 & 0 & 0 & 2 & 0 & 0 & 0 & 0 \\
\hline 23 & 51 & 0 & 0 & 0 & 0 & 12 & 0 & 0 & 0 & 0 \\
\hline 24 & 28 & 0 & 0 & 0 & 0 & 4 & 0 & 0 & 0 & 0 \\
\hline
\end{tabular}

P. zopfin genotype $1(\mathrm{n}=1)$

$P$. zopfii genotype $2(\mathrm{n}=9)$

. blaschkeae $(\mathrm{n}=2$

. zopfii genotype $2(\mathrm{n}=7)$

P. zopfii genotype $2(\mathrm{n}=4)$ 

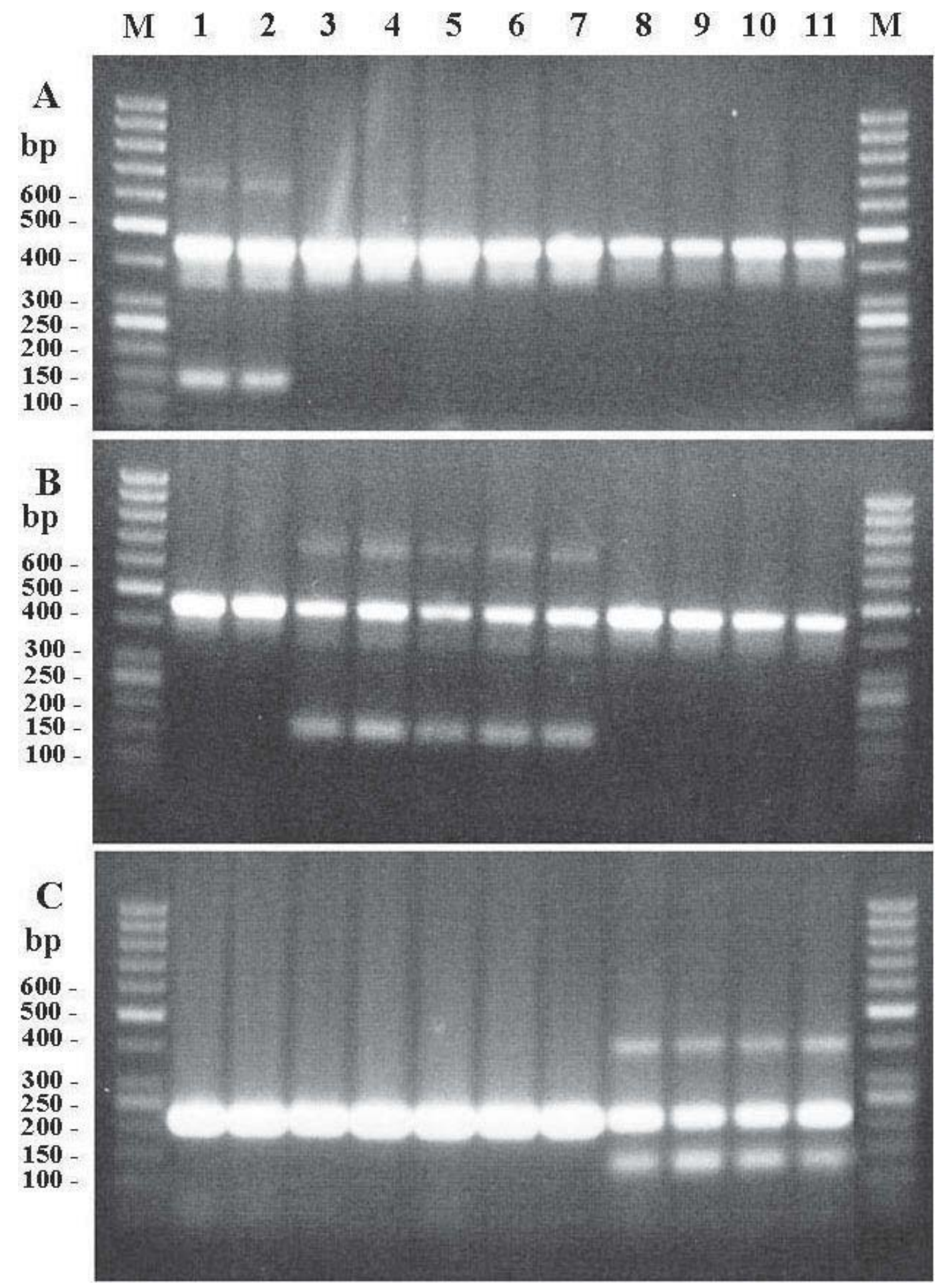

Figure 1. Results of genotype-specific PCR analysis for Prototheca spp. isolates. A) Analysis specific for Prototheca zopfii genotype 1; B) analysis specific for $P$. zopfii genotype 2; C) analysis specific for Prototheca blaschkeae. $\mathrm{M}=$ molecular size marker; lane $1=P$. zopfii genotype 1 SAG 2063 (control strain); lane $2=P$. zopfii genotype 1 strain isolated in the present study; lane $3=P$. zopfii genotype $2219509 / 2$ (control strain); lanes $4-7=P$. zopfii genotype 2 strains isolated in the present study; lane $8=P$. blaschkeae SAG 2064 (control strain); lanes $9-11=P$. blaschkeae strains isolated in the present study. 
ers PZGT 3-IK/f and PZGT 3-IK/R were used, the 213-bp fragment of the internal amplification control was detected in all investigated strains (Figure 1). The presence of $P$. blaschkeae-specific pattern $(126 \mathrm{bp})$ was found in 3 of $108(2.8 \%)$ milk samples and in 7 of 53 $(13.2 \%)$ environmental samples (in particular, in bedding, feces, and drinking water; Table 2).

No significant $(P<0.01)$ correlation was observed $(\rho$ $=0.06$ ) between the percentage of milk and environmental samples positive for Prototheca spp.

The present study represents the first large-scale screening survey aimed at characterizing the diffusion of both P. zopfii genotypes and P. blaschkeae from bovine mastitis milk and cow barn surroundings of Italian dairy herds.

The data reported herein confirmed the role of $P$. zopfii genotype 2 as a major mastitis agent, whereas $P$. blaschkeae was sporadically isolated. Prototheca zopfii genotype 2 strains were isolated from individual milk sampled from cows exhibiting clinical (milk alterations with watery appearance, chronic subacute mastitis with fibrosis), subclinical (absence of milk alterations but increase of SCC) and, rarely, latent mastitis (neither milk alterations nor increase of SCC). On the other hand, the 3 strains of $P$. blaschkeae were isolated from milk produced by one cow with clinical mastitis and from 2 cows with subclinical mastitis (exhibiting only an increase of SCC).

Our data contradict previous hypothesis about the supposed exclusive role of $P$. zopfii genotype 2 as the causative agent of protothecal mastitis (Möller et al., 2007; Osumi et al., 2008) and, in contrast, confirm the study of Marques et al. (2008), supporting the hypothesis that such pathology could be caused by P. blaschkeae, although less frequently. Moreover, we confirmed the status of $P$. zopfii genotype 1 as an environment-related organism, with no involvement in mastitis etiology. However, compared with other reported data (Osumi et al., 2008), its occurrence on Italian dairy herds was surprisingly low.

The isolation of P. zopfii genotype 2 and P. blaschkeae strains (about 85 and $13 \%$ of strains, respectively) from the environment surrounding the cow barn suggests that the potential exposure of cow mammary glands to these yeast-like microalgae through contaminated bedding, feces, and water is considerable. No protothecal contamination of environmental samples was observed in the 6 uninfected herds. However, in view of the absence of any significant $(P<0.01)$ correlation between the prevalence of Prototheca spp. in milk and in environmental samples, the existence of a relationship between udder infection and environmental contamination by Prototheca spp. remains questionable.
In the framework of managing protothecal mastitis outbreaks, the importance of a molecular characterization of Prototheca spp. strains isolated from both individual milk and environmental samples must be stressed. This information is critical to clarify the route of infection for bovine udder in order to set up an effective prophylactic program. Moreover, worldwide, large-scale investigations on the occurrence of different Prototheca species and genotypes may represent the first step to explore the distribution of these etiological agents in different regions and countries.

\section{ACKNOWLEDGMENTS}

Authors thank Gian Luca Belletti (Istituto Zooprofilattico Sperimentale of Lombardia and Emilia Romagna, Piacenza, Italy) for the helpful revision of the manuscript.

\section{REFERENCES}

Buzzini, P., S. Menichetti, C. Pagliuca, C. Viglianisi, E. Branda, and B. Turchetti. 2008a. Antimycotic activity of 4-thioisosteres of flavonoids towards yeast and yeast-like microorganisms. Bioorg. Med. Chem. Lett. 18:3731-3733.

Buzzini, P., B. Turchetti, E. Branda, M. Goretti, M. Amici, P. E. Lagneau, L. Scaccabarozzi, V. Bronzo, and P. Moroni. 2008b. Largescale screening of the in vitro susceptibility of Prototheca zopfii towards polyene antibiotics. Med. Mycol. 46:511-514.

Buzzini, P., B. Turchetti, R. Facelli, R. Baudino, F. Cavarero, L. Mattalia, P. Mosso, and A. Martini. 2004. First large-scale isolation of Prototheca zopfii from milk produced by dairy herds in Italy. Mycopathologia 158:427-430.

Casal, M., and J. Gutierrez. 1983. In vitro activity of ribostamycin against Prototheca spp. Mycopathologia 83:21-23.

Castagna de Vargas, A., A. Lazzari, J. M. Santurio, S. H. Alves, G. Ferreira, and L. C. Kreutz. 1998. Isolation of Prototheca zopfii from a case of bovine mastitis in Brazil. Mycopathologia 142:135-137.

Cunha, L. T., S. M. Pugine, M. R. Silva, E. J. Costa, and M. P. De Melo. 2010. Microbicidal action of indole-3-acetic acid combined with horseradish peroxidase on Prototheca zopfii from bovine mastitis. Mycopathologia 169:99-105.

Furuoka, H., A. Anri, Y. Arita, N. Tuzuki, H. Satoh, and C. Itakura. 1989. Protothecal mastitis in a cow. Jpn. J. Vet. Sci. 51:197199.

Janosi, S., F. Ratz, G. Szigeti, M. Kulcsar, J. Kerenyi, T. Lauko, F. Katona, and G. Huszenicza. 2001. Review of the microbiological, pathological and clinical aspects of bovine mastitis caused by the alga Prototheca zopfii. Vet. Q. 23:58-61.

Kawai, K., K. Shimazaki, H. Higuchi, and H. Nagahata. 2007. Antibacterial activity of bovine lactoferrin hydrolysate against mastitis pathogens and its effect on superoxide production of bovine neutrophils. Zoonoses Public Health 54:160-164.

Lass-Flörl, C., and A. Mayr. 2007. Human protothecosis. Clin. Microbiol. Rev. 20:230-242.

Lagneau, P. E. 1996. First isolation of Prototheca zopfii in bovine mastitis in Belgium. J. Mycol. Med. 6:145-148.

Marques, S., E. Silva, J. Carvalheira, and G. Thompson. 2006. In vitro antimicrobial susceptibility of Prototheca wickerhamii and Prototheca zopfii isolated from bovine mastitis. J. Dairy Sci. 89:4202-4204.

Marques, S., E. Silva, C. Kraft, J. Carvalheira, A. Videira, A. R. Volker, and G. Thompson. 2008. Bovine mastitis associated with Prototheca blaschkeae. J. Clin. Microbiol. 46:1941-1945. 
McDonald, J. S., J. L. Richard, and J. Cheville. 1984. Natural and experimental bovine intramammary infections with Prototheca zopfii. Am. J. Vet. Res. 45:592-595.

Möller, A., U. Truyen, and U. Roesler. 2007. Prototheca zopfii genotype 2. the causative agent of protothecal mastitis? Vet. Microbiol. 120:370-374.

Myers, J. L., and A. D. Well. 2003. Research Design and Statistical Analysis. 2nd ed. Lawrence Erlbaum Associates, Mahwah, NJ.

National Mastitis Council. 1999. Laboratory Handbook on Bovine Mastitis. Revised. National Mastitis Council Inc., Madison, WI.

Osumi, T., Y. Kishimoto, R. Kano, H. Maruyama, M. Onozaki, K. Makimura, T. Ito, K. Matsubara, and A. Hasegawa. 2008. Prototheca zopfii genotypes isolated from cow barns and bovine mastitis in Japan. Vet. Microbiol. 131:419-423.

Pore, R. S. 1973. Selective medium for the isolation of Prototheca. Appl. Microbiol. 26:648-649.

Pore, R. S. 1998. Prototheca Krüger. Pages $883-887$ in The Yeasts: A Taxonomic Study. C. P. Kurtzman and J. W. Fell, ed. Elsevier, New York, NY.

Ribeiro, M. G., M. Rodrigues de Farias, U. Roesler, K. Roth, S. M. Rodigheri, M. A. Ostrowsky, T. Salerno, A. K. Siqueira, and M. C. Fernandes. 2009. Phenotypic and genotypic characterization of Prototheca zopfii in a dog with enteric signs. Res. Vet. Sci. 87:479-481.

Roesler, U., A. Möller, A. Hensel, D. Baumann, and U. Truyen. 2006. Diversity with the current algal species Prototheca zopfii: A proposal for two Prototheca zopfii genotypes and description of a novel species, Prototheca blaschkeae sp. nov. Int. J. Syst. Evol. Microbiol. 56:1419-1425.

Roesler, U., H. Scholz, and A. Hensel. 2003. Emended phenotypic characterization of Prototheca zopfii: A proposal for three biotypes and standards for their identification. Int. J. Syst. Evol. Microbiol. 53:1195-1199.
Salerno, T., M. G. Ribeiro, H. Langoni, A. K. Siqueira, E. O. Costa, P. A. Melville, V. F. Bueno, A. A. Yamamura, U. Roesler, and A. V. Silva. 2010. In vitro algaecide effect of sodium hypochlorite and iodine based antiseptics on Prototheca zopfii strains isolated from bovine milk. Res. Vet. Sci. 88:211-213.

Satoh, K., K. Ooe, H. Nagayama, and K. Makimura. 2010. Prototheca cutis sp. nov., a newly discovered pathogen of protothecosis isolated from inflamed human skin. Int. J. Syst. Evol. Microbiol 60:1236-1240.

Scaccabarozzi, L., B. Turchetti, P. Buzzini, G. Pisoni, L. Bertocchi, N. Arrigoni, P. Boettcher, V. Bronzo, and P. Moroni. 2008. Isolation of Prototheca spp. strains from environmental sources of dairy herds. J. Dairy Sci. 91:3474-3477.

Segal, E., A. A. Padhye, and L. Ajello. 1976. Susceptibility of Prototheca species to antifungal agents. Antimicrob. Agents Chemother. 10:75-79.

Shahan, T. A., and R. S. Pore. 1991. In vitro susceptibility of Prototheca spp. to gentamicin. Antimicrob. Agents Chemother. 35:24342435.

Tortorano, A. M., A. Prigitano, G. Dho, R. Piccinini, V. Daprà, and M. A. Viviani. 2008. In vitro activity of conventional antifungal drugs and natural essences against the yeast-like alga Prototheca. J. Antimicrob. Chemother. 61:1312-1314.

Turchetti, B., P. Buzzini, M. Goretti, E. Branda, G. Diolaiuti, C. D'Agata, C. Smiraglia, and A. Vaughan-Martini. 2008. Psychrophilic yeasts in glacial environments of Alpine glaciers. FEMS Microbiol. Ecol. 63:73-83.

Ueno, R., N. Urano, and M. Suzuki. 2003. Phylogeny of the nonphotosynthetic green micro-algal genus Prototheca (Trebouxiophyceae, Chlorophyta) and related taxa inferred from SSU and LSU ribosomal DNA partial sequence data. FEMS Microbiol. Lett. $223: 275-280$ 